\title{
Effects of casein phosphopeptide amorphous calcium fluoride paste on white spots lesions during orthodontic treatment: one year follow up- tooth mousse gc in white spot during orthodontic treatment
}

\begin{abstract}
The effects of casein phosphopeptide amorphous calcium fluoride phosphate (CPP-ACFP) paste on the remineralization of white spot lesions, evaluated clinically (Nyvad Index) and by standard photography's. The aim of the study was analyses clinically, the effect of Tooth Mouse in orthodontic patients with active white scores. During orthodontic treatment, 12 patients with 134 white spot lesions, using Nyvad criteria and clinical photos were taken in order to confirm the diagnostic criteria. The patients were oriented to apply tooth mouse before going to sleep, after brushing the teeth, with a clean dry finger or cotton tip and leaved for 3 minutes and expectorate but not washed. The Patients were reexamined at three and six months after the mouse use of tooth mosse. Nyvad Scores 1:112 faces $(83,6 \%)$; $2=14$ faces $(10,4 \%) ; 3=6$ faces $(4,5 \%) ; 5=2$ faces $(1,5 \%)$. The active white spots, were more frequent in score 1 , when compared to scores 2,3 and 5 , significant at $1 \%$. Score 2 , had significative differences towards score 5 . The active white spots, were more frequent in score 1 , when compared to scores 2,3 and 5 , significant at $1 \%$. Score 2 , had significative differences towards score 5. After three month, from the 118 white spot lesions, the majority of them inactivated $114(96,61 \%)$, became inactive, while 4 remain active. The scores achieved after three month remain stable till one year evaluation. Tooth mouse, used topically in Brazilian patients, during orthodontic treatment, was effective in remineralizing white spots over one year period.
\end{abstract}

Keywords: casein phosphopeptide amorphous calcium fluoride phosphate paste, orthodontics, white pot lesions, nyvad criteria, remineralization
Volume 2 Issue 2 - 2015

\author{
Groisman S,' Borzino L, ${ }^{2}$ Olival A, ${ }^{2}$ Borzino \\ T,' Corvino $\mathrm{M}^{2}{ }^{2}$ Toledo $\mathrm{M}^{3}$ \\ 'Department of Community Dentistry, Federal University of Rio \\ de Janeiro, Brazil \\ ${ }^{2}$ Federal University of Niteroi, Brazil \\ ${ }^{3}$ Estadual University of Rio de Janeiro, Brazil
}

Correspondence: Groisman S, Department of Community Dentistry, Federal University of Rio de Janeiro, Rua Pinto Guedes 23/302,Tijuca, Brazil, Tel +55219880697/5, Email sonia@dentistas.com.br

Received: March 19, 2015 | Published: April 20, 2015
Abbreviations: CPP-ACFP, casein phosphopeptide amorphous calcium fluoride phosphate; WSL, white spot lesion; ICDAS, international caries detection and assessment system; MB, multibracket

\section{Introduction}

Dentistry in its design as a profession designed to promote and maintain oral health of the population, its main challenge, in Brazil and in the world, the management of dental caries, which still remains a major public health problem in terms prevalence, incidence and severity with which reaches population. ${ }^{1}$ Dentistry has seen great advances in recent decades, concerning the materials and techniques used, with more emphasis on the preventive oral health. ${ }^{2}$ Decalcification or multiple white spot lesion (WSL) development of the enamel surface is by far the most important visible iatrogenic effect of orthodontic multi-bracket (MB) appliance therapy. ${ }^{1-6}$ The incidence is higher in orthodontic MB patients than in nonorthodontic individuals. ${ }^{7}$ White spot lesions are defined as subsurface enamel porosities caused by an imbalance between demineralization and remineralization. When WSLs are located on smooth surfaces they present as milky-white opacities. ${ }^{1,2}$ Demineralization of enamel around brackets can be a very rapid process. ${ }^{1,5}$ It has been observed that these WSLs can disappear ${ }^{8}$ especially in combination with good oral hygiene. ${ }^{9}$ Management of WSLs should involve methods of both preventing demineralization and encouraging the remineralization of existing lesions. In both of these processes, the efficacy of fluoride is well established..$^{10}$ To enhance the natural remineralization by saliva, products providing calcium and phosphate in bioavailable forms have existed since the 1980s when Reynolds et al. ${ }^{11}$ The use of complex composed of amorphous calcium phosphate stabilized by casein fosfopeptidio (CPP -ACP) has proven effective in anti-caries effect in laboratory experiments, in animal studies and human. ${ }^{10}$ The biggest advantages of this compound is related to its potential to penetrated into the body of carious lesions, this mechanism of action is the opposite of the fluorides, where the penetration of ions are confined to the most superficial zone of caries lesion.

The compound of casein is thermodynamically driven to the surfaces of apatite crystals, and ions of $\mathrm{Ca}$ and $\mathrm{P}$ are released and 
deposited in the empty spaces of those crystals $^{3,10,12}$ promoting remineralization. Casein phosphopeptide amorphous calcium phosphate with fluoride (CPP-ACFP) has the same potential actions with the additional benefit of fluoride. ${ }^{13}$ Using CPP-ACFP will remineralize subsurface lesions by forming fluorapatite within the lesion. ${ }^{12}$ Tooth creams, for use supplementary to the normal daily oral hygiene procedure. The remineralization of enamel subsurface lesions by CPP-ACP complexes has been demonstrated in numerous laboratory, animal, and human in situ experimental studies. ${ }^{11,12,14-19}$ Two clinical trials on the remineralization of post orthodontic WSLs by CPP-ACP cream show contrasting finding ${ }^{20,21}$ but clinically, it has been observed that WSLs can disappear. ${ }^{8}$ It has been claimed that the multifactorial anticariogenic mechanism for CPP-ACP has a threefold mode of action:

1. It promotes the remineralization of enamel lesions by maintaining a supersaturated state of the enamel minerals calcium and phosphate in plaque. ${ }^{21}$

2. It delays the formation of biofilm ${ }^{14}$ and inhibits bacterial adhesion to the tooth surface; and

3. It acts as a buffering agent, which may prevent a reduction of $\mathrm{pH}$ in the oral micro-environment. ${ }^{14}$

Casein phosphopeptide amorphous calcium phosphate with fluoride (CPP-ACFP) has the same potential additional benefits of added fluoride. ${ }^{14}$ Using CPP-ACFP will rematerialize subsurface lesions by forming fluorapatite within the lesion. ${ }^{12}$ Therefore, the aim of this study was to investigate the effects of commercially available CPP-ACFP paste (MI-Paste Plus) in vivo, its effect on the remineralization of enamel WSLs, as assessed by Nyvad Criteria, in a randomized controlled clinical trial during a 12-month timeperiod. The null hypothesis was that the use of CPP-ACFP, in vivo, in addition to normal oral hygiene, does not have an effect on(i) the remineralization of subsurface lesions, measured by Nyvad criteria in orthodontic patients with WSLs during fixed orthodontic treatment.

\section{Materials and methods}

During orthodontic treatment, 12patients with 118 white spot lesions, using Nyvad criteria and clinical photos were taken in order to confirm the diagnostic criteria, were select, indicated by one orthodontic dentist (LB), exclusion criteria was not have any systemic disease, no syndromatic abnormalities, no prove or suspected milk protein allergy/or sensitivity, not using at least three months of any medicine that could decrease saliva rate, smoking, not having dental surface with localized visual dentin breakdown after air drying (32) or did not agree in participated in the study.

\section{Subjects and study outline}

Subjects were only enrolled after signed informed consented was obtain from the patient, none of the patients were under 18years old. None of the subjects lived in area where the community water was fluoridated. Subjects complying with the inclusion criteria determined by presence of White spot, were randomly assigned by CT to apply tooth mouse GC (with CPP-ACFP), in all patients that also received oral health instruction and tooth brushing individually. They were orientated to achieve the White spot and to apply the tooth mouse before going to sleep, after brushing the teeth, with a clean dry finger or cotton tip and leaved for 3 minutes and expectorate but not washed, during four weeks. After the first three weeks, the patients were examined and photographed. Monitoring: three, six, nine, and twelve months after the application of mouse tooth, to verify the effectiveness of GC Tooth Mousse on remineralization of active white spots by the clinical register of white spot lesion (WSL), active and inactive lesions, using Nyvad criteria.

\section{Results \\ Descriptive results}

From a total of 12 screened participants, lesions .The patients were reexamined at three and six months. Nyvad Scores 1:112 faces $(83,6 \%) ; 2=14$ faces $(10,4 \%) ; 3=6$ faces $(4,5 \%) ; 5=2$ faces $(1,5 \%)$. The active white spots, were more frequent in score 1 , when compared to scores 2, 3 and 5, significant at $1 \%$. Score 2 , had significative differences towards score 5 . The active white spots, were more frequent in score 1, when compared to scores 2, 3 and 5, significant at $1 \%$. Score 2, had significative differences towards score 5 (Table 1). After three month, from the 134 white spot lesions, the majority of them inactivated $130(97,01 \%)$, became inactive, while 4 remain active. The scores achieved after three month remain stable till one year evaluation (Table 2). Figure $1 \& 2$ shows clinical differences before and after the use of a raging on 4 week uses consecutively tooth mousse in orthodontic treatment, during treatment (Figure 3A \& 3B), and after orthodontic treatment (Figure 4A \& 4B).

Table I White Spots X Nyvad Scores

\begin{tabular}{llllll}
\cline { 2 - 5 } White Spots & Score I & Score 2 & Score 3 & Score 5 \\
\cline { 2 - 2 } & $112(83,6 \%)$ & $14(10,4 \%)$ & $6(4,5 \%)$ & $2(1,5 \%)$ \\
Inactive & $22(16,4 \%)$ & $120(89,6 \%)$ & $128(95,5 \%)$ & $132(98,5 \%)$ & $134(100,0 \%)$ \\
\hline
\end{tabular}

$X^{a}=329,99(P=0.000)$ Significant at $1 \%$

Table 2 Inactivation of white spots after three months period

\begin{tabular}{lllll}
\hline White Spots & Baseline (n) & Baseline (\%) & After 3 Months (n) & After 3 months (\%) \\
\hline Active & 134 & 100 & 11 & $8,2 \%$ \\
Inactive & 0 & & 123 & $91,8 \%$
\end{tabular}

Citation: Groisman S, Borzino L, Olival A, et al. Effects of casein phosphopeptide amorphous calcium fluoride paste on white spots lesions during orthodontic treatment: one year follow up- tooth mousse gc in white spot during orthodontic treatment.J Dent Health Oral Disord Ther. 20I5;2(2):57-60. DOI: I0.15406/jdhodt.2015.02.00043 
$4,50 \%$

\section{Before Tooth Mousse Use}

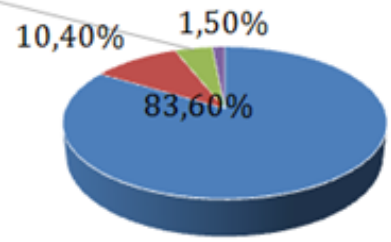

" Active White Spots Score 1 - Active White Spots Score 2

- Active White Spots Score 3 - Active White Spots score 5

Figure I Active lesions (Nyvad classification) sample classification.

\section{active White Spots After six months Tooth Mousse Use}

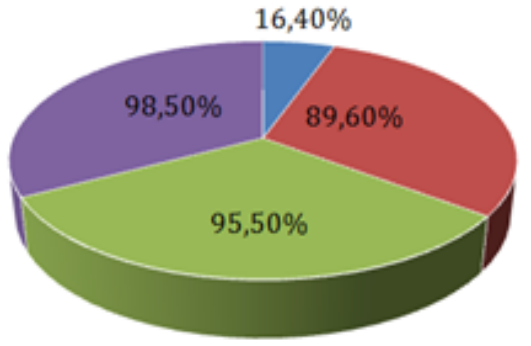

- Inactive White Spots Score 1 - Inactive White Spots Score 2

- Inactive White Spots Score 3 - Inactive White Spots Score 5

Figure 2 Percentage of reminerilazion of white active spots after 4 weeks using tooth mouse every day.

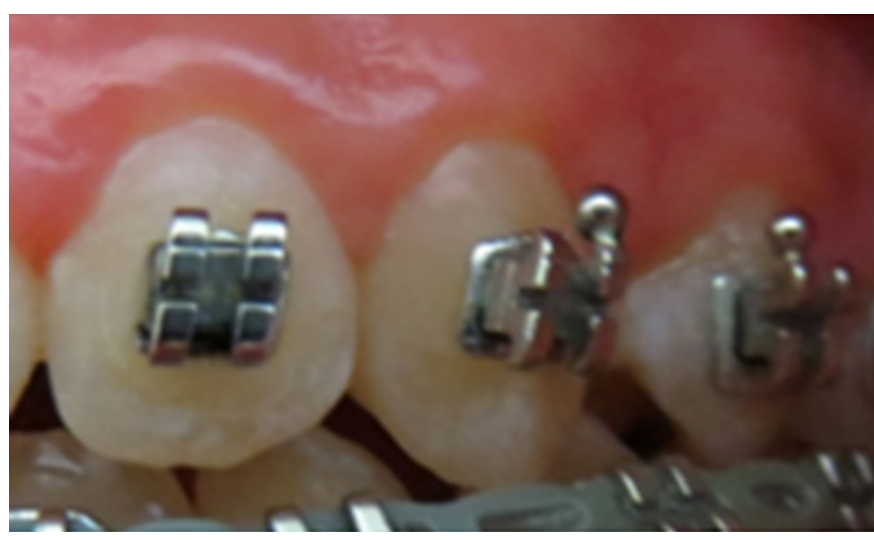

Figure 3 (A) Lucas before tooth mousse use.

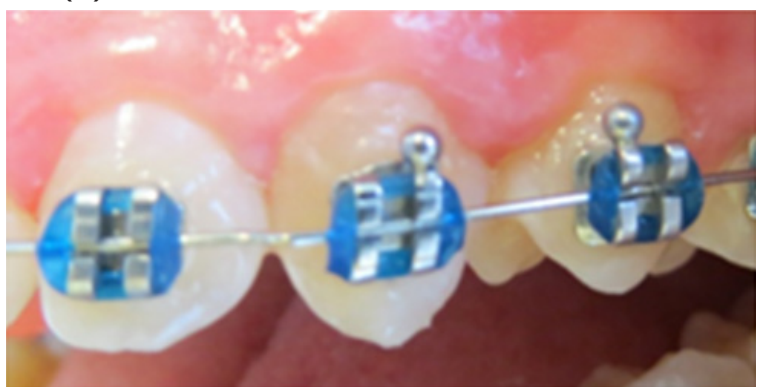

Figure 3 (B) Lucas after three month 4 weeks TM.

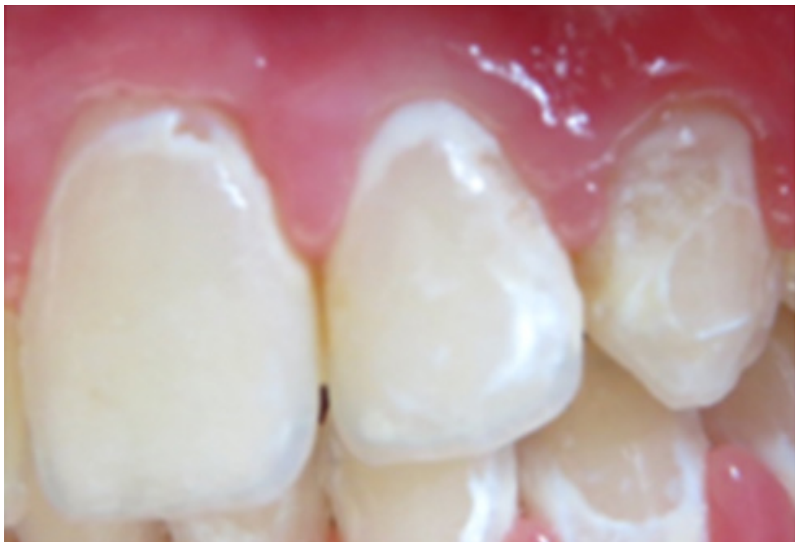

Figure 4 (A) Angelica before tooth mousse.

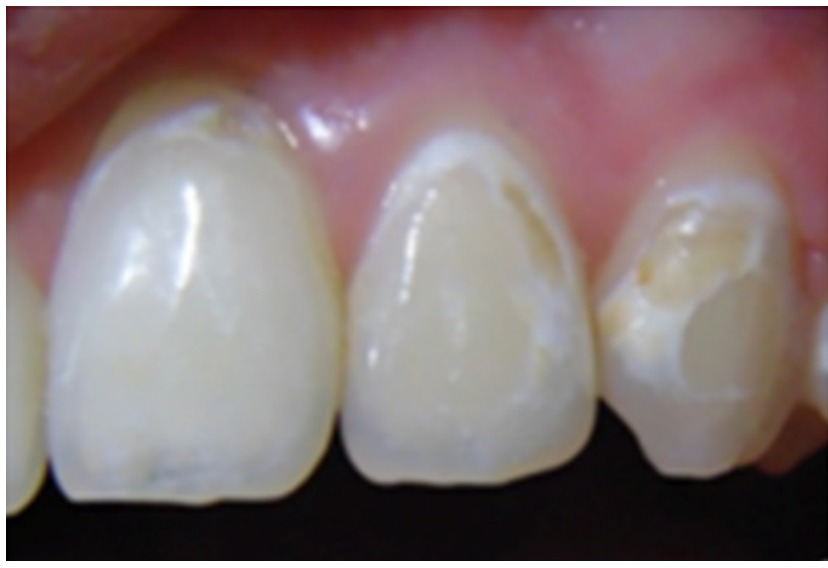

Figure 4 (B) Angelica one year after 4 weeks tooth mousse.

\section{Discussion}

The null hypothesis, which stated that the use of CPP-ACFP in vivo, in addition to normal oral hygiene, does not have an effect on

I. remineralization of subsurface lesions analyzed by Nyvad criteria in orthodontic patients with WSLs during the fixed orthodontic treatment, was rejected.

This contradicts the findings reported by Beerens et al. ${ }^{22}$ that did not observed regression of post orthodontic WSLs and the effect on plaque composition after the use of cream containing CPP-ACP. One fact must be related is that the Beeren ${ }^{22}$ study WLS, was diagnosed by The International Caries Detection and Assessment System $(\text { ICDAS })^{23}$ and by QLF, while in the present study Nyvad Criteria was used but supports the findings of Brochner et al. ${ }^{24}$ who studied the effects of a remineralization cream containing CPP-ACP in the enhanced regression of post orthodontic WSLs and the effect on plaque composition. Brochner et al. ${ }^{24}$ reported a reduction in lesion area of $58 \%$ after only 4 week use. However, the lesions investigated by Brochner et al. ${ }^{24}$ were extremely small in comparison to the lesions studied in Beeren ${ }^{22}$ study, our study, lesion size was not measured. The fact that some studies the lesions did not easily remineralized, the efficacy of these products still require to be proven in long-term clinical studies to ensure that they do indeed protect the WSLs from further demineralization..$^{22}$

Citation: Groisman S, Borzino L, Olival A, et al. Effects of casein phosphopeptide amorphous calcium fluoride paste on white spots lesions during orthodontic treatment: one year follow up- tooth mousse gc in white spot during orthodontic treatment.J Dent Health Oral Disord Ther. 20 I5;2(2):57-60. 


\section{Conclusion}

In conclusion, this clinical trial showed that the use of the commercially available CPP-ACFP paste (MI Paste Plus) was successfully in the remineralization of enamel WSLs during a 12-month follow-up of orthodontic patients, even used only for 4 weeks. The Nyvad scores achieved after 4 weeks remain stable after three month and TILL one year evaluation. Tooth mouse, used topically in Brazilian patients, during orthodontic treatment, was effective in remineralizing white spots over one year period.

\section{Funding}

None.

\section{Acknowledgments}

None.

\section{Conflicts of interest}

The authors declare that there is no conflict of interest.

\section{References}

1. Silva MF, Burgess RC, Sandham HJ, et al. Effects of water-soluble components of cheese on experimental caries in humans. J Dent Res. 1987;66(1):38-41.

2. Krol DM. Dental caries, oral health and pediatricians. Curr Probl Pediatr Adolesc Health Care. 2003;33(8):253-270.

3. Reynolds EC. Casein Phosphopeptide-amorphous Calcium Phosphate: the scientific evidence. Adv Dent Res. 2009;21(1):25-29.

4. Cross KJ, Huq NL, Reynolds EC. Casein phosphopeptides in oral healthchemistry and clinical applications. Curr Pharm Des. 2007;13(8):793800

5. Cai F, Shen P, Morgan MV, et al. Remineralization of enamel subsurface lesions in situ by sugar-free lozenges containing casein phosphopeptideamorphous calcium phosphate. Aust Dent J. 2003;48(4):240-243.

6. Schupbach P, Neeser JR, Golliard M, et al. Incorporation of caseinoglycomacropeptide and caseinophosphopeptide into the salivary pellicle inhibits adherence of mutans streptococci. J Dent Res. 1996;75(10):1779-1788.

7. Azarpazhooh A, Limeback H. Clinical Efficacy of Casein Derivatives: a Systematic review of the Literature. J Am Dent Assoc. 2008;139(7):915924

8. Llena C, Forner L, Baca P. Anticariogenicity of casein phosphopeptideamorphous calcium phosphate: a review of the literature. J Contemp Dent Pract. 2009;10(3):1-9.

9. Cochrane NJ, Saranathan S, Cai F, et al. Enamel subsurface lesion remineralisation with casein phosphopeptide stabilised solutions of calcium, phosphate and fluoride. Caries Res. 2008;42(2):88-97.
10. Reynolds EC, Cai F, Cochrane NJ, et al. Fluoride and casein phosphopeptide-amorphous calcium phosphate. $J$ Dent Res. 2008;87(4):344-348.

11. Reynolds EC, Cai F, Shen $\mathrm{P}$, et al. Retention in plaque and remineralization of enamel lesions by various forms of calcium in a mouthrinse or sugarfree chewing gum. J Dent Res. 2003;82(3):206-211.

12. Reynolds EC. Calcium phosphate-based remineralization systems scientific evidence?. Aust Dent J. 2008;53(3):268-273

13. Reynolds EC, Johnson IH. Effect of milk on caries incidence and bacterial composition of dental plaque in the rat. Arch Oral Biol. 1981;26(5):445451.

14. Rahiotis C, Vougiouklakis G, Eliades G. Characterization of oral films formed in the presence of CPP-ACP agent: An in situ study. $J$ Dent. 2008;36(4):272-280.

15. Shen P, Manton DJ, Cochrane NJ, et al. Effect of added calcium phosphate on enamel remineralization by fluoride in a randomized controlled in situ trial. J Dent. 2011;39(7):518-525.

16. Pulido MT, Wefel JS, Hernandez MM, et al. The Inhibitory Effect of MI Paste, Fluoride and a Combination of both on the Progression of artificial caries-like lesions in enamel. Oper Dent. 2008;33(5):550-555.

17. Bailey DL, Adams GG, Tsao CE, et al. Regression of post-orthodontic lesions by a remineralizing cream. J Dent Res. 2009;88(12):1148-1153.

18. Behnan SM, Arruda AO, Gonzalez-Cabezas C, et al. In vitro evaluation of various treatments to prevent demineralization next to orthodontic brackets. Am J Orthod Dentofacial Orthop. 2010;138(6):712.

19. Nyvad B, Machiulskiene V, Baelum V. Reliability of a new caries diagnostic system differentiating between active and inactive caries lesions. Caries Res. 1999;33(4):252-260.

20. Duckworth RM, Morgan SN, Gilbert RJ. Oral fluoride measurements for estimation of the anti-caries efficacy of fluoride treatments. J Dent Res. 1992;71:836-840

21. Lynch RJ, Navada R, Walia R. Low-levels of fluoride in plaque and saliva and their effects on the demineralisation and remineralisation of enamel; role of fluoride toothpastes. Int Dent J. 2004;54(5S1):304-309.

22. Beerens MW, van der Veen MH, van Beek H, et al. Effects of casein phosphopeptide amorphous calcium fluoride phosphate paste on white spot lesions and dental plaque after orthodontic treatment: a 3-month follow-up. Eur J Oral Sci. 2010;118(6):610-617.

23. Topping GVA, Pitts NB, ICDAS. Detection, assessment, diagnosis and monitoring of caries. Clinical visual caries detection. Monogr Oral Sci. 2009;21:15-41.

24. Brochner A, Christensen C, Kristensen B, et al. Treatment of postorthodontic white spot lesions with casein phosphopeptide-stabilised amorphous calcium phosphate. Clin Oral Investig. 2011;15(3):369-373. 